\title{
Re: A No-Stop Mutation in MAGEB4 is a Possible Cause of Rare $X$-linked Azoospermia and Oligozoospermia in a Consanguineous Turkish Family
}

J Assist Reprod Genet 2017;34:683-694. doi: 10.1007/s10815-017-0900-z.

\section{EDITORIAL COMMENT}

Ten to 15 percent of infertile male patients are diagnosed with azoospermia and in almost 50\% of these patients the etiology of azoospermia is still unknown and defined as idiopathic. Animal models of male infertility has shown that more than 2300 genes were involved in spermatogenesis. Therefore, it is likely that most idiopathic human forms of sperm production failure may have a genetic origin. In this study, the authors investigated mutations causing spermatogenetic failure using whole exome sequencing in a consanguineous Turkish family comprising 2 daughters and 6 sons. Two of male infertile triplets were monozygotic, one of them was cryptozoospermic and one younger infertile brother was azoospermic in semen analysis. Whole exome sequencing showed that all infertile brothers had a homozygous mutation in the melanoma antigen family B4 (MAGEB4) and the mother and fertile two sisters were carriers; the father and two fertile brothers were wild-type that was confirmed via Sanger sequencing. MAGEB4 belongs to a group of genes in the melanoma antigen family with specific expression to germ cells and animal model have shown that MAGEB4 plays a role in germ cell-specific mitosis.

Like TEX11, which is another an X-linked gene, MAGEB4 is another gene that may be responsible for azoospermia in infertile men. Physiological studies on animal models may enable us better understanding of the role of MAGEB4 protein in spermatogenesis.

Emre Bakırcıoğlu, MD

${ }^{\circ}$ Copyright 2017 by the Association of Urological Surgery / Journal of Urological Surgery published by Galenos Publishing House. 